\title{
Sensitive Ratiometric Probing of Tamsulosin Hydrochloride Based On Dual-Emission Fluorescent In Biological Fluids Utilizing Amine-Carbon Quantum Dots And Rhodamine B
}

\author{
Aida Mousavi \\ Iran University of Science and Technology \\ Rouholah Zare-Dorabei ( $\nabla$ zaredorabei@iust.ac.ir) \\ Iran University of Science and Technology \\ Seyed Hossein Mosavi \\ Iran University of Science and Technology
}

\section{Research Article}

Keywords: TMS detection, amine-CQDs, RhB, amine-CQDs/RhB probe, RF sensor, FL quenching

Posted Date: June 8th, 2021

DOI: https://doi.org/10.21203/rs.3.rs-557370/v1

License: (c) (1) This work is licensed under a Creative Commons Attribution 4.0 International License.

Read Full License 


\section{Abstract}

In this work, amine-carbon quantum dots (CQDs)/rhodamine $\mathrm{B}(\mathrm{RhB})$ ratiometric fluorescent (RF) sensor was employed for effective and selective determination of tamsulosin hydrochloride (TMS) based on a dual-emission fluorescence system. Although the function of amine-CQDs is to transfer the specific interaction between TMS and sensor into detectable fluorescence $(\mathrm{FL})$ signals, RhB as a reference unit has been employed to omit internal and external effects. The FL signal was quenched by adding the TMS at $442 \mathrm{~nm}$; nevertheless, it did not change at $569 \mathrm{~nm}$. The material characterization and investigation of the sensing mechanism were done by UV-Vis, fluorimetry, EDS, TEM, FT-IR, and zeta potential analyses. The optimization of $\mathrm{pH}$, the volumetric ratio of CQDs to $\mathrm{RhB}$, and interaction time parameters were carried out by the one-variable-at-a-time (OVAT) method. The quantitative analysis of the concentration of TMS for this RF sensor in a linear range of $0.446-7.083 \mu \mathrm{g} \mathrm{mL}^{-1}$ was obtained $\left(R^{2}=0.9969, n=3\right)$ under optimum conditions. The limit of detection and quantitation values were estimated to be $0.033 \mu \mathrm{g} \mathrm{mL}^{-1}$ and 0.109 $\mu \mathrm{g} \mathrm{mL}^{-1}$, respectively. Repeatability of intra-day and inter-day were less than one percent. This inexpensive RF probe was well applied to determine TMS in biological fluids, and acceptable achievements were obtained.

\section{Introduction}

Tamsulosin hydrochloride (TMS) 5-[(2R)-2-[[2-(2-Ethoxyphenoxy)ethyl]amino]-propyl]-2methoxybenzenesulfonamide hydrochloride, is a class of alpha-1 (a1) adrenergic receptor antagonist family, which is used for monotherapy benign prostate hyperplastic (BPH) ${ }^{1,2}$. These antagonist agents block a1A-receptors in the bladder's neck, and the prostate results in appeasing symptoms of BPH by relaxing the smooth muscle there ${ }^{3}$. Up to now, several analytical techniques have been reported for recognition of TMS, such as high-performance liquid chromatography (HPLC) ${ }^{4}$, liquid chromatographyMS ${ }^{5}$, capillary electrophoresis (CE) ${ }^{6,7}$, electrochemical methods (EC) ${ }^{8}$, UV-vis spectrophotometry ${ }^{9}$, fluorescence spectroscopy $(\mathrm{FL})^{10}$. Chromatographic methods, despite the excellent measurement advantages in trace concentrations, require expensive equipment like CE assay, and toxic solvents; also, EC methods have weak repeatability 11,12 . Thus, developing a rapid and inexpensive method with high accuracy is needed to determine TMS in the biological fluids.

Fluorescent probes have recently become one of the most popular techniques for chemo/bioimaging and chemo/biosensing because the FL method is a low-cost, high-selectivity assay technique compared to radioactive tracers used for biological assays. The ratiometric fluorescent (RF) probes are one of the fluorescent sensors, which have attracted considerable attention because these sensors have higher sensitivity and accuracy than single-signal sensors. In general, an RF probe should be made of two different fluorophores in a sensing system. The RF sensor has a built-in correction factor, eliminating background interferences and environmental effects ${ }^{12-15}$. 
Fluorescent carbon quantum dots (CQDs), as a prominent member of carbon-containing nanomaterials, which researchers take an interest in CQDs over the past decade due to their unequaled properties such as excellent water dispersibility, non-toxicity, superior biocompatibility, easy preparation, inexpensive, resistance to photobleaching and optical stability ${ }^{16-19}$. These extraordinary properties of CQDs have turned them ideal materials in biosensors ${ }^{20}$, light-emitting devices ${ }^{21}$, drug delivery ${ }^{22}$, and imaging ${ }^{23}$. The quasi-spherical CQDs nanoparticles are less than $10 \mathrm{~nm}$ in size and are composed of the core and the shell. The core is constituted of $\mathrm{sp}^{2}$-hybridized carbon clusters, and the shell contains $\mathrm{sp}^{3}$-hybridized carbons, which there are different functional groups on their surface ${ }^{16,24}$.

Herein, amine-CQDs with intense blue FL were synthesized by a one-step hydrothermal method from precursors of EDA and CA. Synthetic amine-CQDs have good water dispersibility, high luminescence, and galore functional groups. In this study, we reported CQDs/RhB as a novel RF probe for the TMS determination. Components amine-CQDs and RhB make up this RF sensor; amine-CQDs act as a receptor and reporter element that have been employed to determine the TMS, while RhB has been used as a reference element due to its optical stability, attractive photophysical properties, and high quantum yield, which reduces interferences caused by environmental factors ${ }^{25,26}$. In the FL spectra of this sensor, two emission peaks are observed, which the peak of amine-CQDs appearing at $442 \mathrm{~nm}$ and the peak of RhB at $569 \mathrm{~nm}$. By increasing TMS concentration to the RF sensor, the emission of amine-CQDs was turned-off while the emission of RhB remained constant. In order to investigate the practical application of this probe in real biofluids samples, it was employed to determine the TMS. To our knowledge, this is the first report to measure TMS by an RF sensor.

\section{Results}

\section{2 - 1 . Characterization}

The morphology of the fabricated amine-CQDs was explained by transmission electron microscopy (TEM). The TEM image displays that CQDs have a spherical shape, and the mean diameter size of these nanoparticles was estimated to be roughly $7.5 \mathrm{~nm}$ (Fig. 1a). To identify the elements of prepared amineCQDs, EDS analysis was carried out. As shown in Fig. 1b, the elements carbon, oxygen, and nitrogen were found. The Fourier transform infrared (FT-IR) spectrum of amine-CQDs is demonstrated in Fig. 1c. The band at $3473 \mathrm{~cm}^{-1}$ belongs to the stretching vibration of $\mathrm{OH}$. Two peaks at $3364 \mathrm{~cm}^{-1}$ and $3301 \mathrm{~cm}^{-1}$ are ascribed to the $\mathrm{NH}$ asymmetric and symmetric stretching vibrations, respectively. The asymmetric and symmetric stretching vibrations of $\mathrm{CH}$ emerged at $2941 \mathrm{~cm}^{-1}$ and $2873 \mathrm{~cm}^{-1}$, respectively. Further, the absorption peak at $2154 \mathrm{~cm}^{-1}$ corresponds to $C=C$ vibration. The vibrations at $1594 \mathrm{~cm}^{-1}$ and 1458 $\mathrm{cm}^{-1}$ are attributed to $\mathrm{C}=\mathrm{O}$ stretching and $\mathrm{NH}$ bending vibrations, respectively. The absorption band at $1363 \mathrm{~cm}^{-1}$ is assigned to $\mathrm{C}-\mathrm{N}$ vibration, and the peak at $710 \mathrm{~cm}^{-1}$ is ascribed to the out-of-plane $\mathrm{NH}$ bending vibration. Furthermore, The FT-IR spectra of RhB and TMS are reported in Figure S1. 
The optical properties of amine-CQDs were investigated by the spectra of UV-Vis and Excitation-Emission photoluminescence (PL). In Figure S2a, two typical absorption peaks are located at $245 \mathrm{~nm}$ and $340 \mathrm{~nm}$, which are attributed to the $\pi \rightarrow \pi^{*}$ transition of $C=C$ and $n \rightarrow \pi^{*}$ transition of $C=0$, respectively. The highest emission intensity is observed under excitation at a wavelength of $365 \mathrm{~nm}$ (Figure S2b). The stability of amine-CQDs was investigated with absorption and FL spectra. The photostability of amineCQDs was assessed after two months of storage; as shown in Figure S3, no noticeable change in FL and UV-Vis intensities is observed. According to Figure S4, the FL intensity of amine-CQDs can remain stable during UV irradiation for thirteen minutes.

\section{2-2. Optimization of experimental parameters}

In order to achieve an excellent response for TMS determination, consequential factors such as $\mathrm{pH}$, the ratio of fluorophores, and interaction time were optimized for the RF sensor. The $\mathrm{pH}$ effect on the $\mathrm{F}_{0} / \mathrm{F}$ value ( $F_{0}$ and $F$ are the $F L$ intensities ratio of the RF sensor in the absence and presence of TMS, where $F_{0}=\left(F_{442} / F_{569}\right)_{0}$ and $\left.F=\left(F_{442} / F_{569}\right)\right)$ was examined by tuning the $B-R$ buffer from 2 to 11 . As shown in Fig. $2 \mathrm{a}$, the $\mathrm{F}_{0} / \mathrm{F}$ value increases by moving to more acidic $\mathrm{pHs}$, while other parameters are considered constant. So, the maximum value is related to $\mathrm{pH}=3$, which is selected as the optimum value because the amine functional group on the CQDs can be protonated by catching $\mathrm{H}^{+}$at acidic media; thus, the active sites of CQDs increase. The second parameter was the volumetric ratio of CQDs to RhB, where pH and other parameters were kept constant. By enhancing the volumetric ratio, the values of $F_{0} / F$ were raised, so according to Fig. $2 b$, the volumetric ratio of 0.2 was chosen as the optimal point because by increasing the concentration of RhB, its interaction with functional groups of CQDs enhances, so lead to reduce the active sites of CQDs, consequently bring about decrease the interaction between CQDs and TMS. The interaction time as the third parameter was optimized, while $\mathrm{pH}=3$, the volumetric ratio of 0.2 , and other items were maintained constant. As illustrated in Fig. 2c, by raising time up to 15 minutes, the $F_{0} / F$ value goes up with a steep slope, and then it becomes approximately invariant. Fifteen minutes was the best interaction time because it takes more time for the analytes to reach the surface of the sensor; for this reason, the interaction time has increased.

\section{2-3. Quenching mechanism}

To construe the process of TMS sensing by an amine-CQDs/RhB sensor, the mechanism between amineCQDs and RhB was examined at first. The emission of conjugated amine-CQDs is reduced compared to free CQDs due to forming a new bond between amine-CQDs and RhB; this causes the Fluorescence resonance energy transfer (FRET) phenomenon to occur, so the emission intensity somewhat decreases ${ }^{27}$. According to Figure S2a, the absorption spectra of amine-CQDs and RhB do not overlap with each other.

Photoinduced electron transfer (PET) can occur when the excited electron is transferred from CQDs (donor) to TMS (acceptor). Thus, for this reason, TMS connects to the carboxylic group of CQDs and 
creates a new interaction. In the following, we investigated the quenching behavior by way of the SternVolmer equation (Eq. 1), zeta potential analysis, and alterations of FT-IR spectrum.

$\mathrm{F}_{0} / \mathrm{F}=\mathrm{K}_{\mathrm{sv}}[\mathrm{Q}]+1$ (Eq. 1)

Where $F_{0}$ and $F$ are the $F L$ intensities ratio of the RF sensor in the absence and presence of TMS, respectively. [Q] represents the concentration of quencher, 'Ksv' is the Stern-Volmer quenching constant ${ }^{28}$. As shown in Fig. 3a, the value of $F_{0} / F$ was plotted for different concentrations of quencher at three temperatures. By increasing temperature, the slope of the curve that represents $\mathrm{K}_{\mathrm{sv}}$ increased. So the $\mathrm{FL}$ quenching might have been happened due to dynamic quenching ${ }^{29,30}$. As it can be seen in Fig. $4 a$, by enhancing the concentration of TMS to this probe, the emission peak of RhB remains constant, while the emission intensity of CQDs reduces. So, TMS could have been detected by CQDs. To further investigate the sensing mechanism, the interaction between amine-CQDs and TMS was studied by zeta potential analysis. As shown in Figure S5, free amine-CQDs have a zeta potential of $-45.8 \mathrm{mV}$, but the zeta potential of them in the presence of TMS makes a dramatic shift to -33 mV, this increases in the surface charge of the amine CQDs after the adding TMS to the solution confirms that functional groups of amine-CQDs have reacted with TMS, so the FL signal reduces. Also, the interaction between amine-CQDs and TMS was examined by FT-IR spectrum. According to Fig. 3b, when TMS was incubated in amine-CQDs solution, the band intensities of $\mathrm{NH}$ stretching vibration reduced to $3364 \mathrm{~nm}$. Not only the absorption peak position of $\mathrm{C}=\mathrm{O}$ has switched from $1594 \mathrm{~nm}$ to $1564 \mathrm{~nm}$, but also the intensity has been lessened. In the FT-IR spectrum of CQDs + TMS, an absorption peak is created in the $1016 \mathrm{~nm}$ region, which is attributed to the $\mathrm{C}-\mathrm{O}$ bond. This band is not observed in the FT-IR spectra of TMS and amine-CQDs. By reducing the intensity of the $\mathrm{C}=\mathrm{O}$ peak and forming the $\mathrm{C}^{-} \mathrm{O}^{-}$band, it can be turned out that a new electrostatic interaction has been established between the $\mathrm{C}^{-\mathrm{O}^{-}}$group of amine-CQDs and the amine-group of TMS, which completes the sensing process.

\section{2-4. Calibration and validation method}

We analyzed the response of the RF probe at different concentrations of TMS under the optimal experimental conditions. By increasing the concentration of TMS to this probe, the signal intensity reduced at $\lambda=442 \mathrm{~nm}$ (Fig. 4a). As shown in Fig. 4b, a good linear relationship could be observed between $\mathrm{F}_{0} / \mathrm{F}$ and the concentration of TMS in the range of $0.446-7.083 \mu \mathrm{g} \mathrm{ml}^{-1}$. The linear regression equation is $F_{0} / F=0.0323\left[C_{a}\right]+0.9888\left(C_{a}\right.$ is the concentration of TMS), and the correlation coefficient is 0.9967 ( $n$ $=3$ ). The detection limit (LOD) and quantitation limit (LOQ) of the proposed method for five tests were estimated to be $0.033 \mathrm{\mu g} \mathrm{ml}^{-1}\left(3 \mathrm{~S}_{\mathrm{b}} / \mathrm{m}\right)$ and $0.109 \mathrm{~g} \mathrm{ml}^{-1}\left(10 \mathrm{~S}_{\mathrm{b}} / \mathrm{m}\right)$, respectively. The comparison between the proposed RF probe and other reported methods to determine TMS is listed in Table 1. This method has a good linear range and a lower LOD than spectrometric methods; this RF probe is cheaper, faster, and more environmentally friendly compared to chromatographic methods. 
In order to evaluate the repeatability of the present recognizing system, we iterated one point by one operator at one place with the same working condition and the same measurement system. Twenty-five experiments were performed in five working days; in this way, five tests were done every day. Thus, as displayed in Fig. 5a, the relative standard deviation (RSD) for intra-day repeatability was calculated to be less than $0.88 \%$, while the RSD for inter-day was computed $0.71 \%$.

Table 1

The comparison of the present study with other reported approaches for TMS detection

\begin{tabular}{|llll|}
\hline Methods & LDR $\left(\mu \mathrm{g} \mathrm{m}^{-1}\right)$ & LOD $\left(\mu \mathrm{g} \mathrm{m}^{-1}\right)$ & Ref. \\
\hline EC & $0.133-445$ & 0.044 & 32 \\
\hline RP-HPLC & $10-50 \times 10^{-3}$ & $0.5 \times 10^{-3}$ & 33 \\
\hline LC-MS/MS & $0.1-19.3 \times 10^{-3}$ & - & 34 \\
\hline UV-VIS & $15-70$ & 1.25 & 35 \\
\hline FL & $0.75-3.50$ & 0.17 & 36 \\
\hline FL & $0.446-8.230$ & 0.033 & This work \\
\hline
\end{tabular}

\section{2-5. Selectivity}

To validate the selectivity of the CQDs/RhB ratiometric probe towards TMS under the optimal conditions, we investigated the response of this sensor to some interfering substances $\left(\mathrm{Al}^{3+}, \mathrm{Ca}^{+2}, \mathrm{Zn}^{2+}, \mathrm{Fe}^{3+}, \mathrm{Cu}^{2+}\right.$, $\mathrm{Mg}^{2+}, \mathrm{K}^{+}, \mathrm{Na}^{+}$, Glucose, Sucrose, L-Cys, L-Leu, and Creatin) in the presence of TMS. As illustrated in Figure $5 b$, among interfering substances, except for copper and iron, all other species are less than $5 \%$ troublemakers on the sensing system. Both copper and iron have a $6.0 \%$ and $5.4 \%$ effect on the sensing process due to the formation of strong interaction with CQDs. The acceptable outcome of selectivity analysis signifies that we can employ this ratiometric sensor to determine TMS in the actual condition.

\section{2-6. Analytical performance of the ratiometric sensor in biofluids}

In order to demonstrate the practical application of the proposed method for TMS recognization in real samples, the experiments were carried out in human urea and serum (AL-Ghadir Hospital, Tehran, Iran). The recoveries of TMS in the human urea were obtained in the range of $94 \%-106 \%$, whereas the RSD range was between $1.1 \%-5.7 \%$. The recovery values in human serum were calculated from $92-102 \%$, whereas RSD was acquired from $2.4-6 \%$. The obtained results in Table 2 indicate that the ratiometric probe is a reliable procedure for analyzing TMS in biofluids. 
Table 2

Results for determination of TMS in real samples

\begin{tabular}{|c|c|c|c|c|}
\hline Sample & Spiked $\left(\mu \mathrm{g} \mathrm{mL} \mathrm{L}^{-1}\right)$ & Found $\pm S D\left(\mu \mathrm{g} \mathrm{mL}^{-1}\right)$ & Recovery $(\%, n=3)$ & $\operatorname{RSD}(\%, \mathrm{n}=3)$ \\
\hline \multirow[t]{5}{*}{ Urea } & 0.658 & $0.702 \pm 0.04$ & 106.7 & 5.7 \\
\hline & 0.987 & $1.022 \pm 0.025$ & 103.5 & 2.4 \\
\hline & 1.425 & $1.356 \pm 0.047$ & 95.1 & 3.5 \\
\hline & 1.864 & $1.753 \pm 0.054$ & 94.1 & 3.1 \\
\hline & 2.193 & $2.210 \pm 0.026$ & 100.8 & 1.1 \\
\hline \multirow[t]{5}{*}{ Serum } & 0.767 & $0.713 \pm 0.025$ & 92.9 & 3.5 \\
\hline & 0.987 & $0.953 \pm 0.023$ & 96.6 & 2.4 \\
\hline & 1.096 & $1.066 \pm 0.049$ & 97.2 & 4.5 \\
\hline & 1.864 & $1.711 \pm 0.046$ & 92.0 & 2.7 \\
\hline & 1.974 & $2.018 \pm 0.121$ & 102.2 & 6.0 \\
\hline
\end{tabular}

\section{Discussion}

Consequently, we developed an RF sensor to determine TMS, which this RF probe is constructed from two agents; amine-CQDs act as the reporter agent that turns specific interactions between the RF sensor and TMS into the recognizable signals, and $\mathrm{RhB}$ as a reference agent was utilized to remove interferences effects on sensing system. By adding TMS to this probe, the emission signal of amine-CQDs is quenched at $442 \mathrm{~nm}$, while the emission signal related to $\mathrm{RhB}$ at $569 \mathrm{~nm}$ has remained constant. The parameters of $\mathrm{pH}$, the volumetric ratio of CQDs to $\mathrm{RhB}$, and interaction time for the RF sensor were optimized using the OVAT method. Therefore, This FL detection method does not require complicated and expensive apparatus, and it displayed high selectivity toward the detection of TMS over various species. According to the comparison made in Table 2, this method has the advantage of higher speed, lower cost, less complexity of the design system compared to reported literature. Besides, the practicability of this RF sensor was successfully validated by sensing TMS in human urea and blood serum samples, which showed satisfactory results that this method can be applied for rapid detection of TAM in biological fluids.

\section{Methods}

\section{4 - 1. Reagents and materials}

The active ingredient of Tamsulosin hydrochloride (TMS) was purchased from Tolid Daru Pharmaceutical Co (Tehran, Iran). The citric acid (CA), Ethylenediamine (EDA), Rhodamine B (RhB), LCysteine (L-Cys), L-Leucine (L-Lue), Creatinin, Sucrose, Glucose, Iron (㽖) chloride, Aluminum chloride, 
Calcium chloride, copper (怄) sulfate, Magnesium chloride, Sodium chloride, Potassium chloride, Zinc sulfate, Acetic acid, Phosphoric acid, Boric acid, and Methanol (Me-OH) with the analytical grade were purchased from Merck Co. (Darmstadt, Germany). Deionized (DI) water with a resistivity of more than 18 $\mathrm{M} \Omega$ has been used in the whole of the experiments.

\section{4 - 2. Apparatus}

The fluorescence measurements were carried out by FL-Ar-2015 fluorescence spectrometer (teifsanje, Iran) and Cary Eclipse fluorescence spectrophotometer (Agilent, USA) with a $1.0 \mathrm{~cm}$ quartz cuvette. The Absorption measurements were performed through a T80 plus spectrophotometer (PG Instruments, Australia(. Transmission electron microscopy (TEM) images were obtained to analyze amine-CQDs morphology using a Philips-EM 208s microscope (FEI Co., USA) at an accelerating voltage of 100kv. Fourier transform infrared (FT-IR) spectra were acquired through Avatar (Thermo, US) in the range of $400-4000 \mathrm{~cm}^{-1}$ by using $\mathrm{KBr}$ pellets. Zeta potential analysis was accomplished using an SZ100 (Horiba, Japan). EDS analysis of amine-CQDs was performed by a MIRA III FE-SEM (Tescan, Czech Republic(. The pHs value was measured and adjusted on a $691 \mathrm{pH}$ meter (Metrohm Co., Swiss). Mixing the solutions was performed on a VORTEX Genius 3 (IKA, Germany).

\section{4 - 3. Synthesis of amine-CQDs}

The amine-CQDs were constructed via a one-step hydrothermal method based on the previous work ${ }^{31}$. Briefly, $3.000 \mathrm{~g} \mathrm{CA}$ and $15 \mathrm{~mL}$ EDA were dissolved in $45 \mathrm{~mL}$ DI water and then poured into a $100 \mathrm{~mL}$ Teflonlined stainless-steel autoclave and treated with $200 \circ \mathrm{c}$ for 5 hours. The resulting brown solution was cooled down to room temperature, then the synthetic solution was filtered through a $0.22 \mu \mathrm{m}$ membrane three times to remove the unreacted species, and finally, the prepared carbon dots were transferred into a dark container and kept at $4{ }^{\circ} \mathrm{C}$.

\section{4-4. General Procedure}

Initially, $1 \mathrm{~mL}$ DI water and $200 \mu \mathrm{L}$ Britton-Robinson (B-R) buffer $(\mathrm{pH}=3)$ were transferred into the cuvette, and then $5 \mu \mathrm{L}$ pure amine-CQDs and $25 \mu \mathrm{L}$ rhodamine-B $\left(20 \mu \mathrm{g} \mathrm{ml}^{-1}\right)$ injected into this solution. After recording the signal of the ratiometric probe, different concentrations of TMS were introduced into the above mixture by micropipette. Ultimately, after incubation for $15 \mathrm{~min}$, the new signal was documented (Fig. 6).

\section{4-5. Sample treatment and ethical statement}

To validate the reliability of the designed RF sensor under real conditions, human urea and blood serum were chosen as actual samples. Human blood serum and urea samples of healthy volunteers were collected in blood collection tubes and clean Falcons by trained staff of AL-Ghadir Hospital (Tehran, Iran). The volunteer gave informed consent with full cognizance of the aim of this project. All experiments and preparation were performed in compliance with the relevant laws and university guidelines as well as with 
the approval by Ethical Committee of Department of Chemistry, Iran University of Science and Technology, Tehran, Iran.

The urea sample was utilized freshly, but the serum sample was stored in the freezer at -20 ॰c. The urea sample was centrifuged at $6000 \mathrm{rpm}$ for $20 \mathrm{~min}$, then $50 \mu \mathrm{L}$ from that was poured separately into five $5 \mathrm{ml}$ volumetric flasks, and different volumes of the standard solution of TMS were added to those flasks, and they were diluted with DI water and B-R buffer $(\mathrm{pH}=3)$, so urea samples just diluted 100 times. The serum sample was thawed gently and was centrifuged at $10000 \mathrm{rpm}$ for $10 \mathrm{~min}$. $250 \mu \mathrm{L}$ of serum sample was transferred to clean Falcon, $250 \mu \mathrm{L} \mathrm{Me}-\mathrm{OH}$ and $5 \mathrm{~mL}$ buffer $\mathrm{B}-\mathrm{R}(\mathrm{pH}=3)$ were added to the Falcon, it vortexed for $5 \mathrm{~min}$, then was centrifuged at $9000 \mathrm{rpm}$ for a quarter. $1 \mathrm{~mL}$ of the centrifuged solution was transferred individually into five $5 \mathrm{ml}$ volumetric flasks, and various amounts of the standard solution of TMS were added to solutions, and dilution was done by DI water.

\section{Declarations}

\section{Competing Interests Statement}

The authors declare no competing financial interest.

\section{Author Contribution:}

Conceptualization, Methodology: A. Mousavi, R. Zare-Dorabei, S.H. Mosavi; Formal Analysis, Writing Original Draft Preparation: A. Mousavi; Supervision, Funding Acquisition, Writing - Review \& Editing: R. Zare-Dorabei.

\section{AUTHOR INFORMATION}

Corresponding Author

*E-mail: zaredorabei@iust.ac.ir; Tel: +98 21 77240646; Fax: +98 2177491204.

ORCID iD Rouholah Zare-Dorabei: https://orcid.org/0000-0002-4106-9878

\section{ACKNOWLEDGMENT}

The author acknowledges financial support from the Iran University of Science and Technology (IUST) \& Iran National Science Foundation (INSF).

\section{Abbreviations}

CQDs, Carbon Quantum Dots; RhB, rhodamine B; TMS, Tamsulosin hydrochloride; BPH, benign prostate hyperplastic FRET, fluorescence resonance energy transfer; PET, Photoinduced electron transfer; OVAT, One-variable-At-a-Time; DI, Deionized; B-R, Britton-Robinson; L-Cys, L-Cysteine; L-Leu, L-Leucine; EDA, Ethylendiamin; CA, Citric acid; Me-OH, Methanol FL, Fluorescence; RF, ratiometric Fluorescence; $\mathrm{CE}$, 
Capillary Electrophoresis; HPLC, High-Performance Liquid Chromatography; MS, Mass Spectrometry; EDS, Energy-dispersive X-ray spectroscopy; UV-vis, Ultraviolet; EC, Electrochemical; TEM, Transmission electron microscopy; FT-IR, Fourier Transform Infrared; LDR, Linear Dynamic Range; LOD, Detection Limit; LOQ; Quantitation Limit.

\section{References}

1. Ramakrishna, N. V. S. et al. Rapid, simple and highly sensitive LC-ESI-MS/MS method for the quantification of tamsulosin in human plasma. Biomed. Chromatogr. 19, 709-719 (2005).

2. Rezk, M. R., Tantawy, M. A., Wadie, M. \& Weshahy, S. A. Smart spectrophotometric assessment of tamsulosin hydrochloride and tadalafil in their new pharmaceutical formulation for treatment of benign prostatic hyperplasia and erectile dysfunction. Spectrochim. Acta - Part A Mol. Biomol. Spectrosc. 227, 117547 (2020).

3. Rezk, M. R., Abdel-Moety, E. M., Wadie, M. \& Tantawy, M. A. Stability assessment of tamsulosin and tadalafil co-formulated in capsules by two validated chromatographic methods. J. Sep. Sci. (2020). doi:10.1002/jssc.202000975

4. Kumari, R., Dash, P. P., Lal, V. K., Mishra, A. \& Murthy, P. N. RP - HPLC method for the estimation of Tamsulosin Hydrochloride in Tablet Dosage Form. Indian J. Pharm. Sci. 72, 785-787 (2010).

5. Agarwal, S. et al. Simultaneous determination of tamsulosin and dutasteride in human plasma by LC-MS-MS. Chromatographia 67, 893-903 (2008).

6. Van Eeckhaut, A. \& Michotte, Y. Chiral separation of cetirizine by capillary electrophoresis. Electrophoresis 27, 2376-2385 (2006).

7. Silvestri, R. et al. Anti-HIV-1 activity of pyrryl aryl sulfone (PAS) derivatives: Synthesis and SAR studies of novel esters and amides at the position 2 of the pyrrole nucleus. Farmaco 59, 201-210 (2004).

8. Özkan, S. A., Uslu, B. \& Aboul-Enein, H. Y. Voltammetric investigation of Tamsulosin. Talanta 61, 147-156 (2003).

9. Gowri Sankar, D., Hemant Kumar, T. \& Vamsi Krishna, M. UV spectrophotometric determination of duloxetine hydrochloride. Asian J. Chem. 21, 4951-4953 (2009).

10. Karasakal, A. \& Ulu, S. T. Validated spectrofluorimetric method for the determination of tamsulosin in spiked human urine, pure and pharmaceutical preparations. Luminescence 29, 239-242 (2014).

11. Hatamluyi, B. \& Es'haghi, Z. Electrochemical biosensing platform based on molecularly imprinted polymer reinforced by $\mathrm{ZnO}$-graphene capped quantum dots for 6-mercaptopurine detection. Electrochim. Acta 283, 1170-1177 (2018).

12. Chen, Z. et al. A fluorescence switch sensor for 6-mercaptopurine detection based on gold nanoparticles stabilized by biomacromolecule. Biosens. Bioelectron. 41, 844-847 (2013).

13. Lakowicz, J. R. Principles of fluorescence spectroscopy. Princ. Fluoresc. Spectrosc. 1-954 (2006). doi:10.1007/978-0-387-46312-4 
14. Sun, J., Mei, H. \& Gao, F. Ratiometric detection of copper ions and alkaline phosphatase activity based on semiconducting polymer dots assembled with rhodamine B hydrazide. Biosens. Bioelectron. 91, 70-75 (2017).

15. Hao, J. et al. Ratiometric fluorescent detection of $\mathrm{Cu} 2+$ with carbon dots chelated Eu-based metalorganic frameworks. Sensors Actuators, B Chem. 245, 641-647 (2017).

16. Chen, J. et al. A fluorescent biosensor for cardiac biomarker myoglobin detection based on carbon dots and deoxyribonuclease l-aided target recycling signal amplification. RSC Adv. 9, 4463-4468 (2019).

17. Chowdhuri, A. R., Singh, T., Ghosh, S. K. \& Sahu, S. K. Carbon Dots Embedded Magnetic Nanoparticles @Chitosan @Metal Organic Framework as a Nanoprobe for pH Sensitive Targeted Anticancer Drug Delivery. ACS Appl. Mater. Interfaces 8, 16573-16583 (2016).

18. Kalaiyarasan, G., Veerapandian, M., Jebamercy, G., Balamurugan, K. \& Joseph, J. AmygdalinFunctionalized Carbon Quantum Dots for Probing $\beta$-Glucosidase Activity for Cancer Diagnosis and Therapeutics. ACS Biomater. Sci. Eng. 5, 3089-3099 (2019).

19. Qin, S. J. \& Yan, B. Dual-emissive ratiometric fluorescent probe based on Eu3+/C-dots@MOF hybrids for the biomarker diaminotoluene sensing. Sensors Actuators, B Chem. 272, 510-517 (2018).

20. Kalaiyarasan, G. \& Joseph, J. Cholesterol derived carbon quantum dots as fluorescence probe for the specific detection of hemoglobin in diluted human blood samples. Mater. Sci. Eng. C 94, 580-586 (2019).

21. Zhou, Z., Wang, Z., Tang, Y., Zheng, Y. \& Wang, Q. Optical detection of anthrax biomarkers in an aqueous medium: the combination of carbon quantum dots and europium ions within alginate hydrogels. J. Mater. Sci. 54, 2526-2534 (2019).

22. Mehta, V. N., Chettiar, S. S., Bhamore, J. R., Kailasa, S. K. \& Patel, R. M. Green Synthetic Approach for Synthesis of Fluorescent Carbon Dots for Lisinopril Drug Delivery System and their Confirmations in the Cells. J. Fluoresc. 27, 111-124 (2017).

23. Zhang, M. et al. A hydrothermal route to multicolor luminescent carbon dots from adenosine disodium triphosphate for bioimaging. Mater. Sci. Eng. C 76, 1146-1153 (2017).

24. Liu, M. L. et al. Anthrax biomarker: An ultrasensitive fluorescent ratiometry of dipicolinic acid by using terbium(III)-modified carbon dots. Talanta 191, 443-448 (2019).

25. Liang, Y. C. et al. A ratiometric fluorescent nanoprobe based on quenched carbon dots-rhodamine $B$ for selective detection of L-cysteine. J. Alloys Compd. 788, 615-622 (2019).

26. Mchedlov-Petrossyan, N. O., Vodolazkaya, N. A. \& Doroshenko, A. O. Ionic Equilibria of Fluorophores in Organized Solutions: The Influence of Micellar Microenvironment on Protolytic and Photophysical Properties of Rhodamine B. J. Fluoresc. 13, 235-248 (2003).

27. Mandani, S., Sharma, B., Dey, D. \& Sarma, T. K. White light emission by controlled mixing of carbon dots and rhodamine $\mathrm{B}$ for applications in optical thermometry and selective Fe $3+$ detection. RSC Adv. 6, 84599-84603 (2016). 
28. Nemati, F., Hosseini, M., Zare-Dorabei, R., Salehnia, F. \& Ganjali, M. R. Fluorescent turn on sensing of Caffeine in food sample based on sulfur-doped carbon quantum dots and optimization of process parameters through response surface methodology. Sensors Actuators, B Chem. 273, 25-34 (2018).

29. Kumar, H. M. S. et al. Analysis of fluorescence quenching of new indole derivative by aniline using Stern-Volmer plots. J. Lumin. 116, 35-42 (2006).

30. Nemati, F., Hosseini, M., Zare-Dorabei, R. \& Ganjali, M. R. Sensitive recognition of ethion in food samples using turn-on fluorescence $\mathrm{N}$ and $\mathrm{S}$ co-doped graphene quantum dots. Anal. Methods 10, 1760-1766 (2018).

31. Mousavi, A., Zare-Dorabei, R. \& Mosavi, S. H. A novel hybrid fluorescence probe sensor based on metal-organic framework@carbon quantum dots for the highly selective detection of 6mercaptopurine. Anal. Methods 12, 5397-5406 (2020).

32. Lonappan, L., Issac, S., Joseph, R., Thomas, D. \& Kumar, K. G. Electrochemical studies of tamsulosin hydrochloride using multiwalled carbon nanotube-modified glassy carbon sensor. Micro Nano Lett. 6 , 867-870 (2011).

33. Thimmaraju, M. K., Rao, V., Hemanth, K. \& Siddartha Kumar, P. RP HPLC Method for the determination of Tamsulosin in bulk and Pharmaceutical formulations. J. Appl. Pharm. Sci. 1, 177-180 (2011).

34. Keski-Rahkonen, P. et al. Determination of tamsulosin in human aqueous humor and serum by liquid chromatography-electrospray ionization tandem mass spectrometry. J. Pharm. Biomed. Anal. 43, 606-612 (2007).

35. El-Henawee, M. M., Abd El-Hay, S. S., Attala, K. \& Eissa, M. S. Smart UV spectrophotometric methods based on simple mathematical filtration and classical methods for the simultaneous determination of tamsulosin and solifenacin: A comparative study of efficacy and spectral resolution. Spectrochim. Acta - Part A Mol. Biomol. Spectrosc. 247, 119151 (2021).

36. El-Kimary, E. I., Khamis, E. F., Belal, S. F. \& Abdel Moneim, M. M. Enhanced spectrofluorimetric determination of two novel combination therapies for the treatment of benign prostatic hyperplasia containing tamsulosin hydrochloride. Luminescence 33, 771-779 (2018).

\section{Figures}



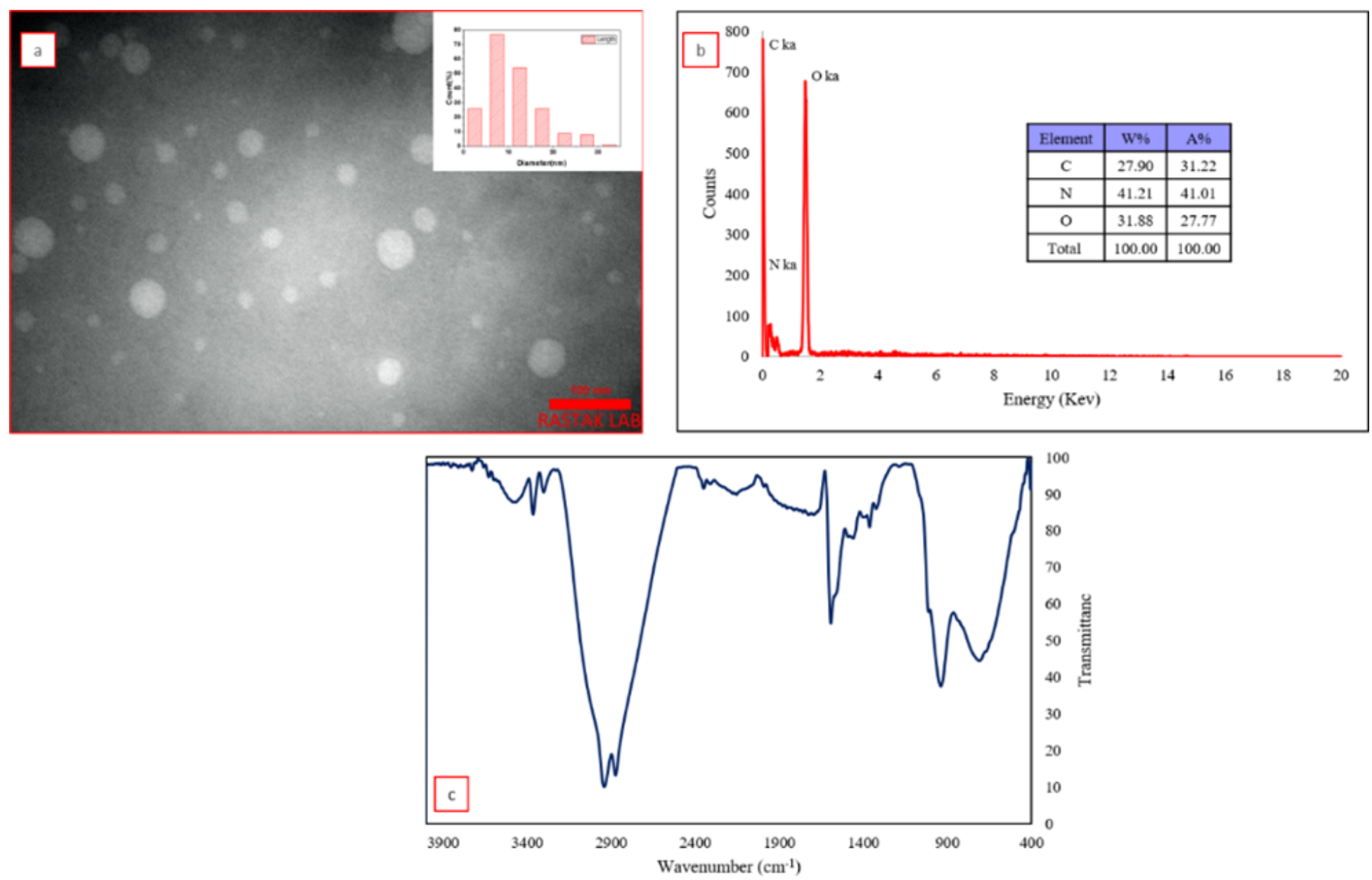

Figure 1

(a) TEM image and particle size distribution, (b) EDS analysis, and (c) FT-IR spectrum of amine-CQDs 

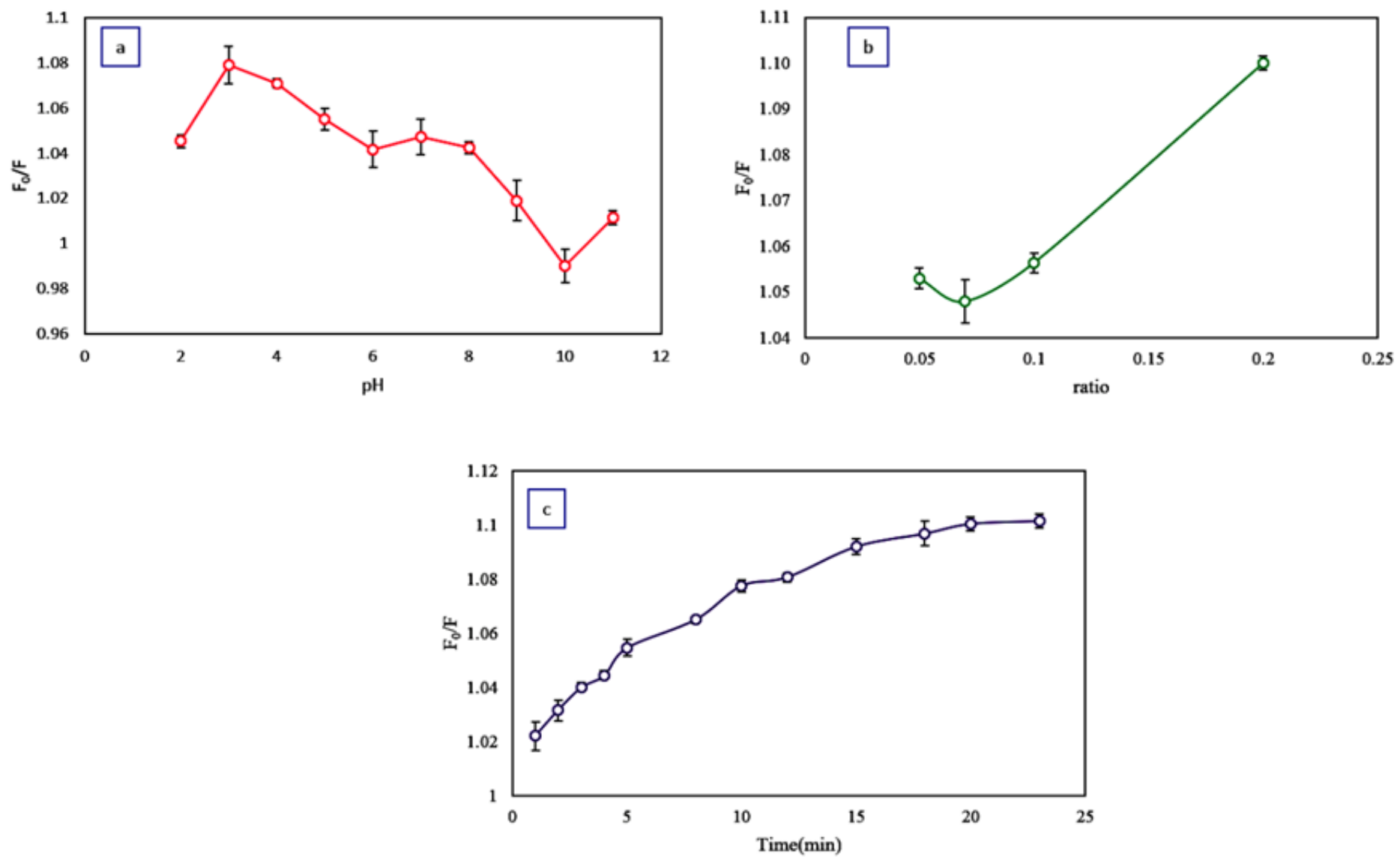

Figure 2

Effect of (a) $\mathrm{pH}(\mathrm{pH}=2-11$, and CTMS $=4.2 \mu \mathrm{g} \mathrm{ml}-1)$, (b) volumetric ratio (ratio= 0.05-0.2, $\mathrm{pH}=3$, and $\mathrm{CTMS}=4.2 \mu \mathrm{g} \mathrm{ml}-1)$, and (c) interaction time $(\mathrm{t}=0-23 \mathrm{~min}, \mathrm{pH}=3$, ratio=0.2, and $\mathrm{CTMS}=4.2 \mu \mathrm{g} \mathrm{ml}-1)$. Error bars represent the standard deviation from three repetitions

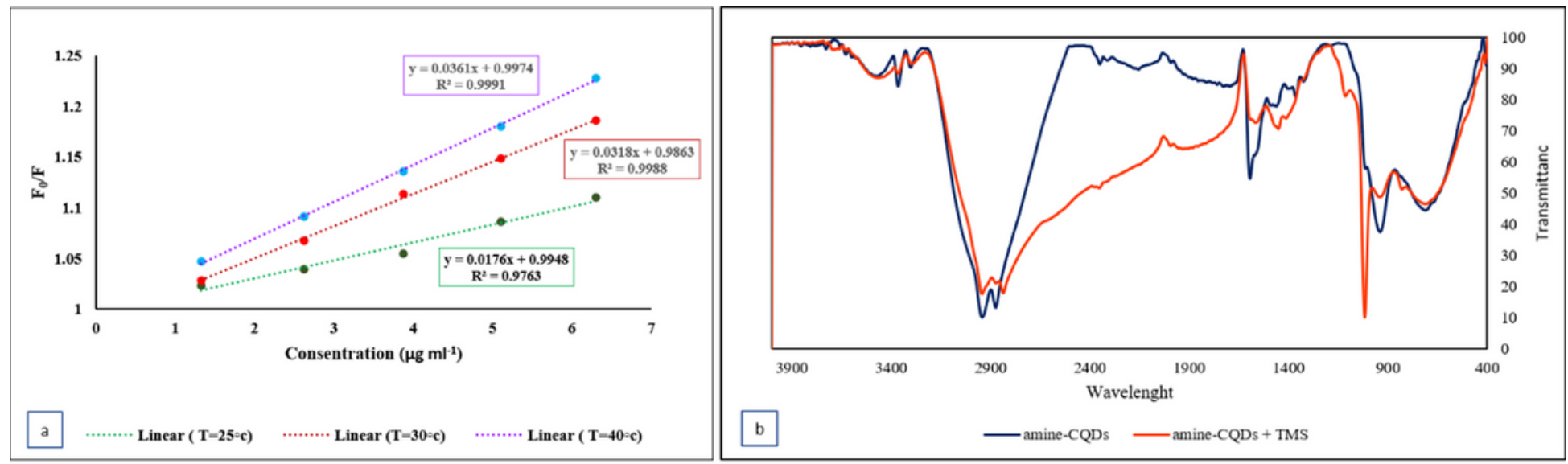

Figure 3 
(a) The Stern-Volmer curves of TMS at the temperatures of $25 \circ \mathrm{c}, 30 \circ \mathrm{c}$, and $40 \circ \mathrm{c}$, (b) FT-IR of amine-CQDs (navy color), and amine-CQDs with TMS (red color)
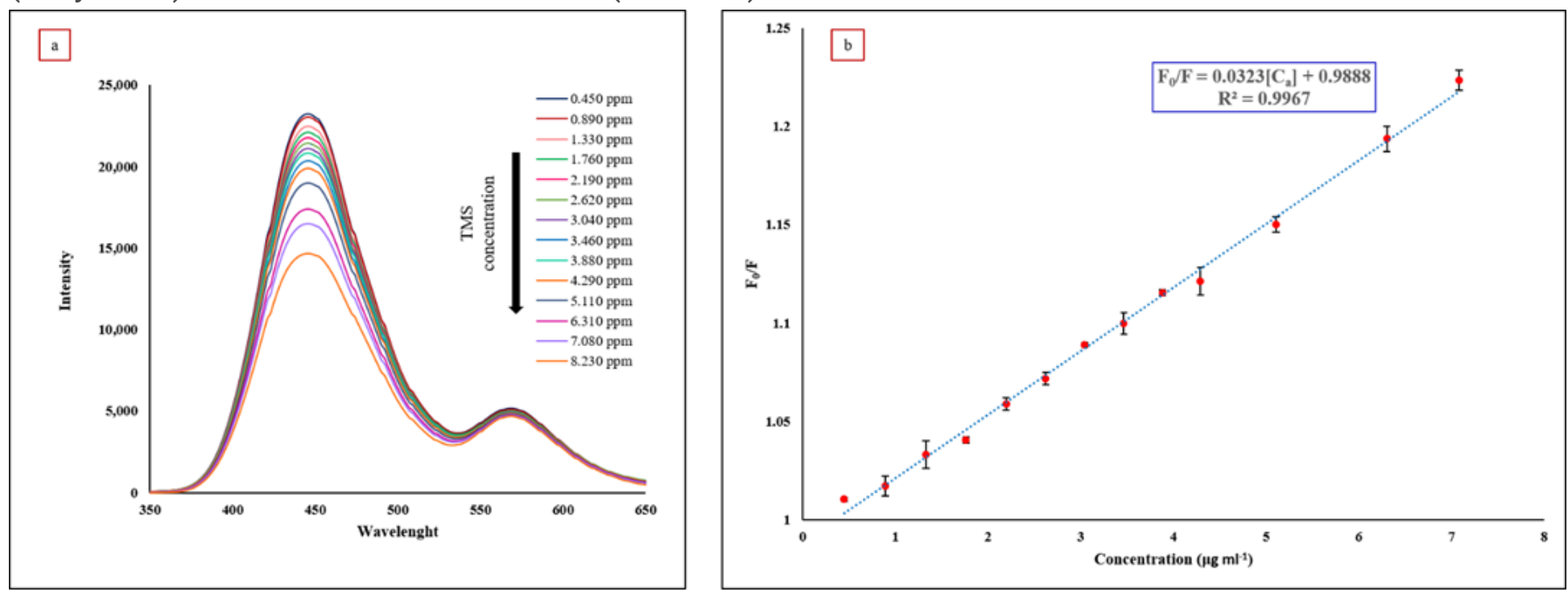

\section{Figure 4}

(a) FL emission spectra of CQD/RhB sensor in the presence of different concentrations of TMS, and (b) linear calibration plot in different concentrations of $\mathrm{TMS}(\mathrm{pH}=3$, volumetric ratio $=0.2$, and time $=15 \mathrm{~min})$. Error bars represent the standard deviation from three repetitions
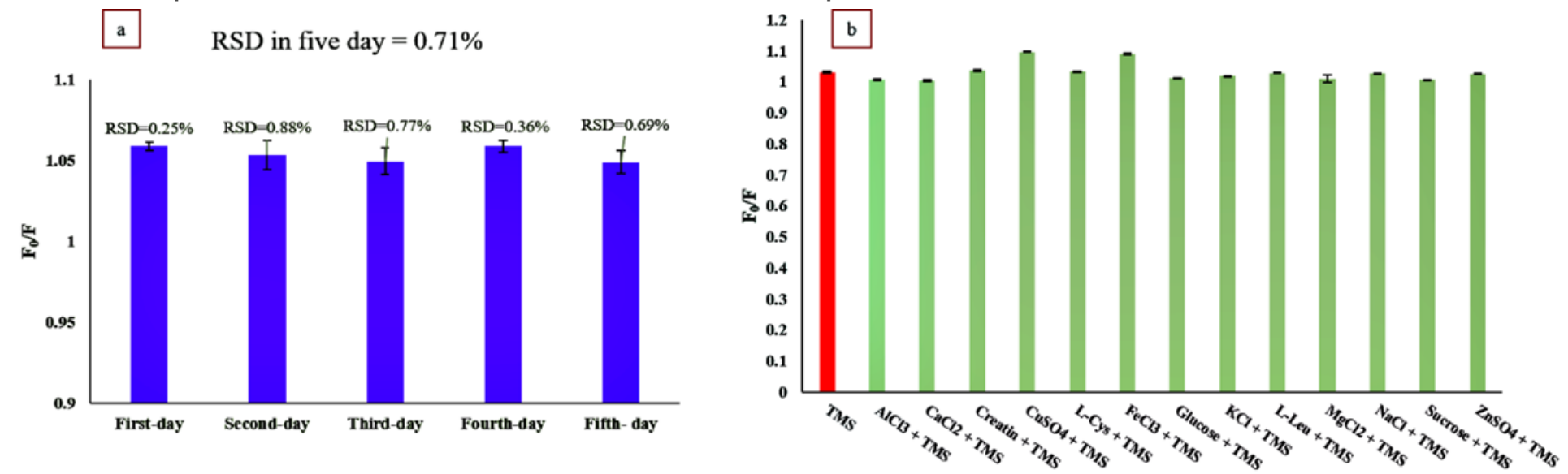

Figure 5

(a) Repeatability of RF sensor in five days (Five testes for each day), and (b) The influence of a few foreign species on the sensing system (CTMS, CL-Cys, and CL-Lus $=0.660 \mu \mathrm{g} \mathrm{ml-1,Cglucose,} \mathrm{Csucaros,}$ CCreatinin, $\mathrm{CCuSO} 4$ and $\mathrm{CFeCl} 3=3.3 \mu \mathrm{g} \mathrm{ml}-1, \mathrm{CNaCl}, \mathrm{CKCL}, \mathrm{CCaCl} 2, \mathrm{CMgCl} 2$ and $\mathrm{CZnSo} 4=6.6 \mu \mathrm{g} \mathrm{ml}-1$ ). Error bars represent the standard deviation from three repetitions of tests under optimal conditions $(\mathrm{pH}=3$, volumetric ratio $=0.2$, and time $=15 \mathrm{~min}$ ) 

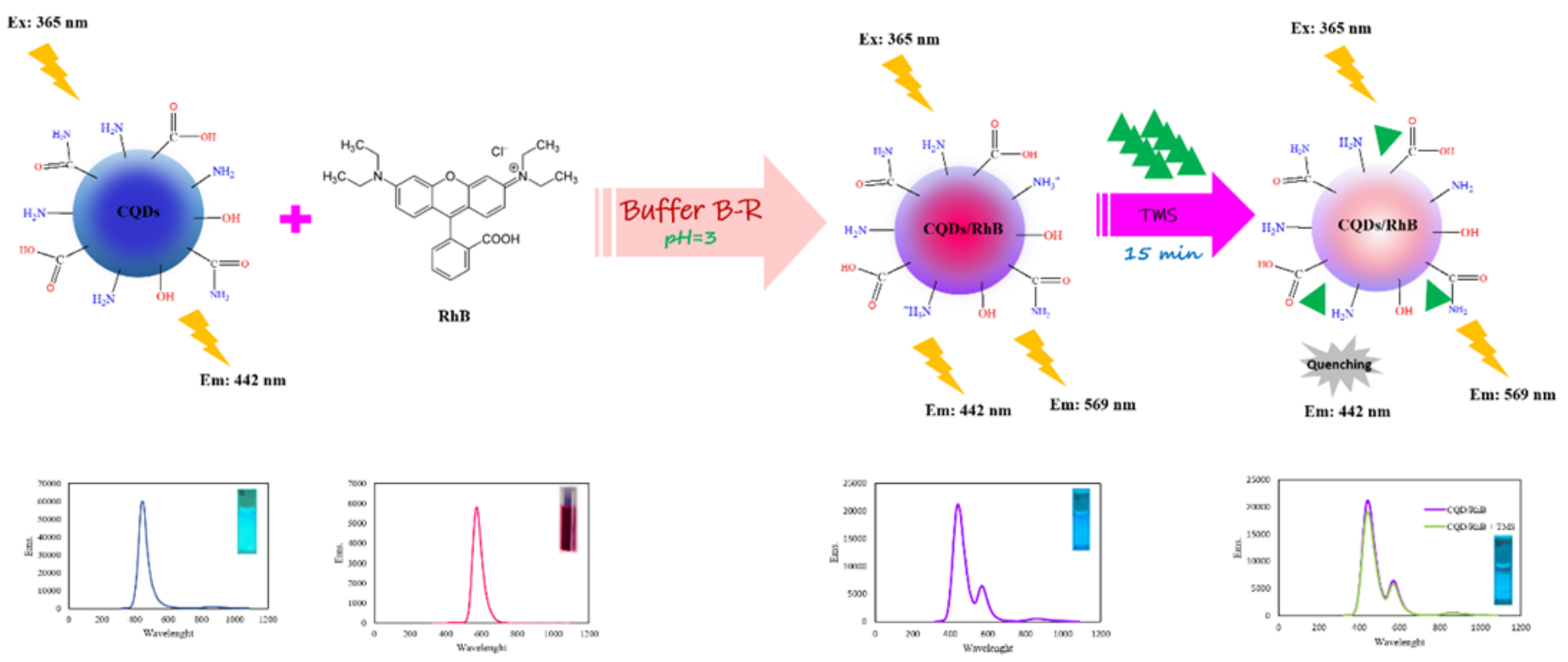

Figure 6

The process of sensing TMS using amine-CQDs/RhB RF probe

\section{Supplementary Files}

This is a list of supplementary files associated with this preprint. Click to download.

- Supplementarydata.pdf 